\title{
Mitteilungen der DMG
}

Gynäkologische Endokrinologie 2021 · 19:88-91

https://doi.org/10.1007/s10304-020-00371-6

(c) Springer Medizin Verlag GmbH, ein Teil von Springer Nature 2021

\section{Herausgeber}

Deutsche Menopause Gesellschaft e.V.

Dr. med. Katrin Schaudig (V.i.S. d. P.)

Praxis für Gynäkologische Endokrinologie, Hormone

Hamburg am Gynaekologicum

Altonaer Straße 59

20357 Hamburg

schaudig@hormone-hamburg.de

www.menopause-gesellschaft.de
Deutsche Menopause Gesellschaft e.V.
Redaktion

Dr. med. Katrin Schaudig

schaudig@hormone-hamburg.de

\section{Jahrestagung der Deutschen Menopause Gesellschaft e.V.}

\section{Menopause und danach - die ganze Frau im Blick}

Welche individuellen Risiken müssen bei der Betreuung von Frauen in der Lebensmitte mit Blick auf eine möglichst lange und gesunde „zweite Lebenshälfte" beachtet werden? Was muss neben den Fragestellungen rund um eine Hormonersatztherapie (HRT) bedacht werden, wenn man Frauen optimal beraten will, um sie gut für die Postmenopause vorzubereiten?

Wie gewohnt fand die Deutsche Menopause Gesellschaft e. V. (DMG) auch dieses Mal auf ihrer Jahrestagung am 13. und 14. November 2020 wieder Antworten auf spannende Fragen. Bei der Themenauswahl der Veranstaltung mit dem Motto „Menopause und danach - die ganze Frau im Blick" ging es darum, nicht nur die hormonellen Aspekte der Menopause und der Zeit danach, sondern auch das Älterwerden per se mit allen seinen körperlichen und psychologischen Facetten zu beleuchten.

Die Tagung, die erstmals unter der Leitung der neuen DMG-Präsidentin Dr. Katrin Schaudig sowie ihrer Co-Kongresspräsidentin Dr. Anneliese Schwenkhagen, beide Hamburg, stattfand, musste sich dieses Jahr jedoch einer großen Herausforderung stellen: Aufgrund der Corona-Pandemie hatten sich die Verantwortlichen entschieden, den Kongress komplett online durchzuführen. Dass dies sehr gut funktionierte und der Kongress den bisherigen an Qualität und Wissensvermittlung in nichts nachstand, zeigte die hohe Teilnehmerzahl von rund 400.

Kleiner Wermutstropfen: Aufgrund der räumlichen Trennung konnte weder das 25. Jubiläum der DMG noch das 2500. Mitglied gebührend gefeiert werden. Mit so vielen Mitgliedern bleibt die DMG weiterhin die größte Menopausegesellschaft der Welt.

\section{Praxis und Wissenschaft}

Auch in diesem Jahr stießen die inzwischen fest zum Programm gehörenden und beliebten Workshops auf großes Interesse. Das Gleiche galt auch für die neu ins Leben gerufenen und schnell ausgebuchten Early bird-Sessions am Samstagmorgen, bei denen man sich mit Expert ${ }^{*}$ innen bei einem Kaffee in kleiner Runde zu verschiedenen Themen austauschen konnte.
Wie gewohnt fand auch bei der virtuellen Kongressvariante die Vortragssession „Neues aus der Wissenschaft“ statt, deren Teilnehmer sich mit spannenden neuen Forschungsergebnissen um Auszeichnungen für hervorragende Beiträge bewarben. Den ersten, mit $1250 €$ dotierten Platz belegte Livia Nakhostin, Bern/Schweiz für ihre Arbeit mit dem Thema „Impact of menopausal hormone therapy on colorectal cancer risk - a systematic review“. Den zweiten Preis und $750 €$ erhielt Katharina Brück, Köln, und den dritten sowie $500 €$ Annika vom Scheidt, Hamburg.

Einige der Abstracts finden Sie in dieser Ausgabe von Gynäkologische Endokrinologie

\section{Ehrenmitglied}

Nachdem im vergangenen Jahr em. Prof. Dr. Herbert Kuhl, Aschaffenburg, die Ehrenmitgliedschaft erhielt, wurde diese Ehre nun em. Prof. Dr. Dr. Hermann P. G. Schneider, Keitum/Sylt, zuteil. Damit würdigte die DMG seine langjährigen Verdienste für die Gesellschaft, an deren Gründung vor 25 Jahren Schneider maßgeblich beteiligt war und deren Geschicke er auch in den Folgejahren erheblich geprägt hat.

\section{Back to the future: HRT in der Prävention bei postmeno- pausalen Frauen}

Für eine Reihe von Erkrankungen kann man von einer Primärprävention durch eine HRT ausgehen (Prof. Dr. Petra Stute, Bern/Schweiz). So ergeben sich positive Effekte nicht nur auf menopausale Symptome, sondern auch auf die Knochen-, die Herz- und die kognitive Gesundheit, auf die Gewichtsstabilisierung und die Prävention von Diabetes mellitus Typ 2, auf Haut und Haare und insgesamt auf ein längeres Überleben. Kritisch sind ein erhöhtes Brustkrebsrisiko bei einer kombinierten und länger angewendeten HRT sowie ein erhöhtes VTE-/ Apoplexrisiko bei einer oralen Östrogengabe.

\section{Volkskrankheit Adipositas}

Zum Thema Adipositas und aktuelle COVID-19-Pandemie gilt, dass starkes Übergewicht ein entscheidender Risikofaktor für schwere und letale Krankheitsverläufe ist (Prof. Dr. Matthias Blüher, Leipzig). Generell sinkt mit steigendem Körpergewicht die Lebenserwartung und über 60 Erkrankungen sind mit Adipositas assoziiert. Nach entsprechender Diagnostik und psychosozialer Evaluation kann in vielen Fällen eine Kombination aus Lebensstil-Interventionen und medikamen- 
töser Therapie zu einer Gewichtsreduktion führen, manchmal sind chirurgische Verfahren (bariatrische Operationen) notwendig.

Gerade bei extremer Adipositas ist die chirurgische Therapie die einzige anhaltend effektive Maßnahme (Prof. Dr. Thomas P. Hüttl, München). Damit kann im Regelfall das angestrebte individuelle Therapieziel erreicht und die Lebenserwartung des/der Betroffenen erhöht werden. Die Wahl für ein bestimmtes Verfahren ist von Faktoren wie BMI, Alter, Geschlecht, Komorbiditäten, Adhärenz und Beruf abhängig. Im Anschluss an Operation und Gewichtsverlust sollten plastische Korrekturen sowie eine engmaschige Nachbetreuung Bestandteil des Gesamtbehandlungskonzeptes sein.

Ein BMI über der 50. Percentile ist bei Jugendlichen mit einer erhöhten kardiovaskulären Mortalität assoziiert. Übergewicht und besonders eine Adipositas im mittleren Alter verkürzen die Lebenszeit (Prof. Dr. Birgit-C. Zyriax, Hamburg). Auch metabolisch gesunde Adipöse haben im Follow-up ein höheres Mortalitätsrisiko. Eine Reihe von Faktoren (z. B. Rauchen, Vorerkrankungen) beeinflussen den $\mathrm{Zu}$ sammenhang zwischen BMI und Mortalität. Aber: Nach einer Krankheitsmanifestation bzw. bei kritisch Kranken kann eine Adipositas das Überleben erhöhen.

\section{Never ending story Gestagen: wofür, welches und wieviel?}

Gestagene sind zur Therapie von Blutungsstörungen und Endometriumhyperplasien sowie zur Endometriumprotektion bei einer HRT entscheidend (Prof. Dr. Thomas Römer, Köln). Bei der Wahl des Gestagens ist im Zusammenhang mit dem Endometrium seine transformatorische Wirkung ausschlaggebend - hier sind z.B. MPA und Dienogest vorteilhafter. Die Dosis sowie das ausgewählte Präparat richten sich u.a. nach dem östrogenabhängigen Proliferationseffekt, der Art der Blutungsstörungen und möglichen Begleiterkrankungen (z. B. Endometriose).

Auch bzgl. des Einflusses auf das Zentrale Nervensystem (ZNS) muss die Gestagenkomponente einer HRT sorgfältig ausgewählt werden. Dem Progesteron etwa wird eine neuroprotektive Wirkung nachgesagt (Prof. Dr. Joseph Neulen, Aachen). Ungünstig scheint das MPA zu sein. Zudem ist unter neuroprotektiven Aspekten eine LangzeitHRT ( $>10$ Jahre) ratsam. Zu beachten ist die Kontraindikation Gehirntumore (insbesondere Meningeome).

Nach wie vor gilt, dass bei einer kombinierten HRT ein erhöhtes Brustkrebsrisiko besteht (Prof. Dr. Dr. Mueck, Tübingen \& Beijing/China). Dies betrifft insbesondere die kontinuierlich kombinierte HRT, in geringerem Maß die sequentiell kombinierte. Das geringste Risiko weist die Estogen-Monotherapie auf. Die Risikoerhöhung hängt von der Dauer der Therapie ab. Zudem unterscheiden sich bzgl. verschiedener Risiken und Effekte auch die Gestagen-Typen, hier scheinen mikronisiertes Progesteron und Dydrogesteron Vorteile aufzuweisen. Künftig müssen Risikofaktoren für Brustkrebs sowie die Mechanismen zur Karzinomentstehung besser identifiziert werden, wie etwa die mögliche beschleunigte Proliferation durch Gestagene.

Eine orale Östrogengabe geht mit einem erhöhten VTE- und Apoplexrisiko (Prof. Stute) einher. Zur Vermeidung von VTEs ist mikronisiertes Progesteron wohl ein günstiger Partner bei der kombinierten HRT, die in Deutschland bei der HRT nicht verfügbaren Norpregnane scheinen besonders ungünstig zu sein. Bei Pregnanen ist bzgl. der Risiken die Unterscheidung verschiedener Typen wichtig. Gleiches gilt auch bei der Vermeidung von ATEs. Bei Tibolon zeigt sich für den Apoplex ein höheres Risiko bei älteren Patientinnen, nicht aber bei jüngeren oder HRT-Starterinnen.

\section{Individuelle Risiken der Patientin in der Lebensmitte - was ist für die Zukunft relevant?}

Bei der Beratung von Patientinnen in der Lebensmitte müssen individuelle Risiken für verschiedene Erkrankungen eruiert werden, um sie für die nächsten Jahrzehnte möglichst optimal vorzubereiten und gegebenenfalls präventiv tätig zu werden. Für das Osteoporoserisiko sollte man neben Faktoren wie Genetik und anamnestische Phasen des Östrogenmangels auch auf Begleiterkrankungen wie Diabetes mellitus, Z.n. Mammakarzinom oder andere chronische Erkrankungen, wie Colitis Ulcerosa, achten, bei denen es zu einer verringerten Knochenstruktur und somit einem erhöhtem Frakturrisiko kommen kann - auch bedingt durch Medikamente wie Aromatasehemmer oder Kortison (Prof. Dr. Peyman Hadji, Frankfurt am Main).

Die erhöhte Prävalenz des metabolischen Syndroms in der Postmenopause erklärt sich u. a. durch die hiermit verbundenen hormonellen Veränderungen, hat aber selbstverständlich auch genetische Ursachen und ist eng verknüpft mit einer Zunahme des BMI und einer Änderung der Körperkomposition (PD Dr. Susanne Hahn, Düsseldorf). Das metabolische Syndrom erhöht nicht nur das Risiko für kardiovaskuläre Erkrankungen, es hat u. a. auch negative Effekte auf das Brustkrebsrisiko und klimakterische Beschwerden. Dem kann man vor allem mit einer Gewichtsabnahme durch Lifestyle-Änderungen entgegenwirken.

Bei kardiovaskulären Krankheiten gilt ebenfalls, dass deren Inzidenz aufgrund verschiedener Risikofaktoren bei Frauen mit dem Alter zunimmt (Prof. Dr. Eberhard Windler, Hamburg). Um dieses Risiko zu verringern, sollte ein gesunder Lebensstil an erster Stelle stehen inklusive einem Rauchstopp. Reicht dies zur Prävention nicht aus, ist unbedingt die medikamentöse Behandlung der Teilaspekte eines metabolischen Syndroms (wie Hypertonus, Dyslipidämie, Diabetes mellitus) anzustreben.

Ein Gentest zur Erfassung genetisch determinierter Karzinomrisiken erfordert eine intensive medizinische und ethische Aufklärung, auch zu den individuellen Konsequenzen bei einer möglichen genetischen Vorbelastung, etwa bei einer BRCA-Mutation (Dr. Usha Peters, Hamburg). Hier ist es für die Betroffenen wichtig zu wissen, welche Maßnahmen zur Risikominimierung relevant sind, wie die Intensivierung von bildgebenden Verfahren zur Früherkennung, aber auch prophylaktische Operationen.

\section{Generation Silver-Sex}

Wenn Paare in die Jahre kommen, wird die Kommunikation über sexuelle Probleme oft als Herausforderung erlebt (Dr. Markus Valk, Wesel). Zur ärztlichen Aufgabe gehört es, die Sexualität selbstverständlich anzusprechen, keine Normierungen zu übertragen und die Paarkommunikation zu fördern. Darüber hinaus sollten Funktionsstörungen erfragt und behandelt werden.

Testosteron kann laut der Leitlinie zu Peri- und Postmenopause bei Frauen mit Libidoverlust erwogen werden, wenn eine HRT nicht wirksam ist, allerdings sollte auf den „No-Label-Use“ hingewiesen werden (Dr. Anneliese Schwenkhagen, Hamburg). Da es immer noch keine speziell für Frauen konzipierte zugelassene Testosteron-Präparate auf dem Markt gibt, muss man sich mit Individualrezepturen behelfen. In der Praxis gibt es gute Erfahrungen mit einem transdermalen Testosterongel $(0,2 \%)$. Für die Therapie der Dysbalance zwischen sexueller Hemmung und sexueller Erregung kommen verschiedene Substanzen infrage. In den USA in dieser Indikation zugelassen sind beispielsweise Flibanserin und Bremelanotid. Studiendaten gibt es auch zu Bupropion, das in Deutschland aber nur als Antidepressivum zugelassen ist. 
Zu möglichen Kombination von Wirkstoffen laufen aktuell verschiedene Studien.

Mögliche sexuelle Probleme des Mannes sollten ebenfalls angesprochen werden (Dr. Viola Kürbitz, Westerstede). Eine erektile Dysfunktion oder eine Ejakulatio praecox können den Patienten erheblich belasten und negative Auswirkungen auf die Partnerschaft haben. Auch hier gilt es, das Paar zu motivieren, sich Hilfe zu suchen.

\section{Im Dialog mit der Patientin}

Der Umgang mit Ängsten bei der Kommunikation mit Patient*innen kommt in der täglichen Routine oft zu kurz. Hier sollte immer zwischen der emotionalen und der fachlichen Gesprächsebene unterschieden werden (Prof. Dr. Dipl. Theol. Matthias Volkenandt, München). Gezieltes Nachfragen („Wovor haben Sie am meisten Angst?") und Zuhören sind elementar. Patient ${ }^{*}$ innen fühlen sich am besten verstanden, wenn sie nicht nur Ratschläge erhalten, sondern in ihren Ängsten ernst genommen werden.

Bzgl. der Kommunikation zu einer HRT ist die Aufklärung der Patientinnen zu möglichen Risiken der Therapie wichtig (Dr. Katrin Schaudig, Hamburg). Dabei sollten auch hier erst die Emotionen, dann die Fakten besprochen werden. Die wichtigsten Risiken einer HRT (Brustkrebs- und vaskuläres Risiko) müssen mit dem Leidensdruck der Patientin sowie den zusätzlichen Nutzen, die sie möglicherweise durch eine HRT hat (u. a. positiver Effekt auf Osteoporose, Diabetes mellitus etc.) ins richtige Verhältnis gesetzt werden. Hierzu gehört auch die Aufklärung über Lifestyle-Faktoren, die für die Risiken und Nutzen eine entscheidende Rolle spielen (Sport, Alkohol, Ernährung, Gewicht etc.).

\section{Altwerden - wie geht das?}

Das Thema des „Altwerdens“ wurde aus sehr unterschiedlichen Blickwinkeln beleuchtet. Hoch interessant ist der Zusammenhang zwischen Telomeren, Stoffwechsel und dem Altern (Prof. Dr. Henriette Kirchner, Lübeck). So verkürzen sich die Telomere nach jeder Zellteilung, zu kurze Telomere induzieren Apoptose, den Stopp der Zellteilung und die Seneszenz (Alterungsprozesse). Viele altersbedingte metabolische Erkrankungen sind mit verkürzten Telomeren assoziiert und ihre Länge ist wiederum proportional zur Mortalität. Und auch wenn die Telomerlänge nicht alles erklären kann, spielen oxidativer, nutritiver und allgemeiner Stress sowie Inflammation eine große Rolle bei metabolischen Erkrankungen und Telomerlänge.

Für den (eigenen) Umgang mit dem Altwerden ist es aus psychiatrischer Sicht ratsam, sich der damit verbundenen physischen und psychischen Defizite bewusst zu sein und diesen gezielt entgegenzuwirken, z. B. durch die Bereitschaft, die eigene Komfortzone zu verlassen, sowohl in geistiger und in emotionaler, aber auch in physischer Sicht (Prof. Dr. Josef Aldenhoff, Hamburg). Beispielsweise ist für die Aktivierung neuer neuronaler Netzwerke in jedem Alter wichtig, neue Dinge zu erlernen, etwa eine Sprache oder ein Instrument.

Einen interessanten Blick über den Tellerrand bot der Vortrag zu der öffentlichen Wahrnehmung von Frauen über 50 in den Medien (Prof. Dr. Elizabeth Prommer, Rostock). Hier ist bemerkenswert, dass Frauen in dieser Altersgruppe in Kino- und Fernsehfilmen deutlich unterrepräsentiert sind. Auch gibt es einen Altersgap, wenn Frauen vorkommen, dann als junge Frauen; ab dem Alter von 30 Jahren kommen Frauen sukzessive seltener vor. Und: Männer erklären die Welt. Sie sind Experten (auch in der Medizin), GameshowModeratoren, Journalisten und Sprecher. In der Corona-Berichterstattung etwa kommen auf eine Frau vier Männer. Dies sollte Anlass zu breitem gesellschaftlichen Diskurs sein und wurde auch von den Tagungsteilnehmerinnen lebhaft diskutiert.

\section{HRT-Update - was Sie jetzt wissen müssen!}

Weitere Neuheit im Programm der DMG-Tagung war eine Session, in der zu verschiedenen Themen die wichtigsten Publikationen des zurückliegenden Jahres vorgestellt wurden - ein gelungener Abschluss des Kongresses.

Quelle: Jahrestagung der Deutschen Menopause Gesellschaft e.V.; OnlineKongress, 13./14. November 2020

Anne Göttenauer

Dipl.-Biologin

anne.goettenauer@unitybox.de

DMG- und Kongresspräsidentin:

Dr. Katrin Schaudig, Praxis für Gynäkologische Endokrinologie, HORMONE HAMBURG am Gynaekologicum, Hamburg

Kongresspräsidentin:

Dr. Anneliese Schwenkhagen, Praxis für Gynäkologische Endokrinologie, HORMONE HAMBURG am Gynaekologicum, Hamburg

\section{Ankündigungen 2021}

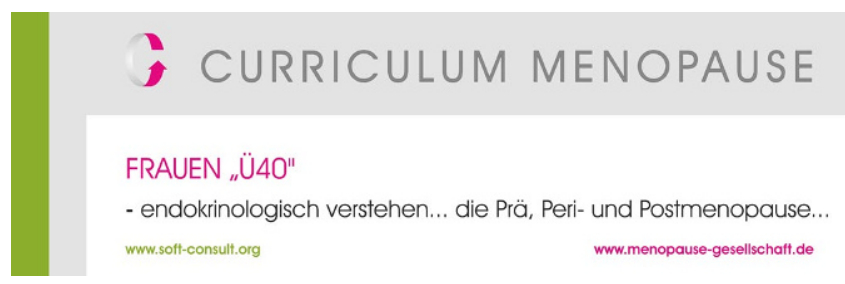

\section{Frauen „Ü40“ endokrinologisch verstehen ...die Prä-, Peri- und Postmenopause ...}

Curriculum Modul I, „Basiswissen Endokrinologie“

19.-20.03.2021, Online

03.-04.12.2021, Hamburg

Curriculum Modul II, ,W'Was sollten Frauenärzte/Innen aus anderen Fachgebieten wissen?"

05.-06.11.2021, Online

\section{Jahrestagung DMG e.V.}

12.-13.11.2021, Frankfurt am Main

Kongresspräsident: Prof. Dr. Joseph Neulen, Aachen

\section{Mitgliederversammlung DMG e.v.}

\subsubsection{1, Frankfurt am Main}

Fragen Sie die Experten - Telefonische Hotline für DMG Mitglieder

Den DMG Mitgliedern steht an jedem ersten und dritten Donnerstag im Monat die kostenlose Telefon-Hotline „Fragen Sie die Experten“ zur Verfügung, bei der wir gerne Ihre Fragen rund um die Menopause beantworten. Das Telefonat ist für Sie gebührenfrei. Weitere Informationen finden Sie im internen Mitgliederbereich der Website oder Sie wenden sich an unsere Geschäftsstelle! 
Für weitere Informationen wenden Sie sich bitte an:

Geschäftsstelle Deutsche Menopause Gesellschaft e.V.

Anne Becker

Weißdornweg 17

D-35041 Marburg/Lahn

Tel.: +49 (0)6420 - 329486

info-dmg@email.de

www.menopause-gesellschaft.de 